\title{
Applied Study on Image Retrievals based on Statistics Projection Algorithm and Robert Algorithm
}

\author{
Xiaofei Zheng ${ }^{1}$, Zhe Gao ${ }^{2}$ and Enping Liu ${ }^{1, *}$ \\ ${ }^{1}$ Institute of Scientific and Technical Information. CATAS, 571737, Danzhou Hainan, China \\ 2 Editorial Department of Journal, Hainan University, Haikou 570228, China
}

Received: 11 Apr. 2013, Revised: 12 Aug. 2013, Accepted: 14 Aug. 2013

Published online: 1 Mar. 2014

\begin{abstract}
In this paper, image retrieval method based on statistics and projection is presented. At first, images are transferred from the RGB color model to the HSI color model, and then images are segmented into sub-images (or blocks) and extract the mainhue and mainintensity in these sub-images. Images's mainhue and mainintensity are projected onto the horizontal and vertical directions, respectively, and four projective histograms were obtained. Finally, the first three central moments are taken as feature as to compute the similarity between images.
\end{abstract}

Keywords: Applied Study, Image Retrievals, Statistics Projection Algorithm, Robert Algorithm, main hue, main intensity, projective histogram

\section{Introduction}

Content-based image retrieval (Content-based Image Retrieval, referred to as CBIR) is the use of visual features of the image, such as color, texture, shape, match the query image and database, return similar images to the user. Color is the most significant feature of the visual image, is also the main perceptual features of people to identify the image, and has received extensive attention and research of [1] in image retrieval. Swain and Ballard [2] proposed the global color histogram, histogram intersection - L1 metric method, [2] color histogram as the similarity measure. Color histogram is the statistics of pixel in an image shows the times, can only reflect the frequency of different color image value, can not reflect the distribution of the relationship between color space, the image retrieval accuracy is not high.

The author puts forward the following ideas retrieval algorithm, image texture and color statistics based on projection: firstly the image is transformed from RGB model to HSI model, extraction of image texture extraction, Robert algorithm, and then the image is divided into blocks, the characteristics of main color block are respectively projected on the horizontal and vertical directions, 2 projection histograms projection histogram, using the first three moments and texture to compute similarity of images.

\section{Colour Model}

Search in the use of color image, select the appropriate color model is especially important for search results. Commonly used color model are: RGB color model, namely the use of R (red), G (green), B (blue) color to represent the color, RGB color model is widely used in display system, but the visual perception of RGB color model and people do not correspond, similar in color similar to that of the RGB model does not reflect the color itself, in the use of RGB color model to retrieve images are RGB color model conversion to other color space.

Because the visual system of HSI color model with the most close to, is a suitable for human resolution model [4]. In HIS model, $\mathrm{H}$ said the tone (Hue), $\mathrm{S}$ represents the color saturation (Saturation), I denote brightness or intensity (Intensity). For any $3 \mathrm{R}, \mathrm{G}, \mathrm{B}$ value in the range of $[0,1]$, can be converted to [5] HSI color model by the following formula

$$
I=\frac{1}{3}(R+G+B)
$$

\footnotetext{
*Corresponding author e-mail: yjlep@163.com
} 


$$
\begin{gathered}
S=1-\frac{3}{R+G+B} \min (R, G, B) \\
H=\left\{\begin{array}{l}
\arccos \frac{(R-G)+(R-B)}{2 \sqrt{(R-G)^{2}+(R-B)(G-B)}}, G \geq B ; \\
2 \pi-\arccos \frac{(R-G)+(R-B)}{2 \sqrt{(R-G)^{2}+(R-B)(G-B)}}, G<B ;
\end{array}\right.
\end{gathered}
$$

The range of calculated $I$ and $S$ formula for $[0,1]$, in the range $H$ of $[0,2 \pi]$, in order to make $H$ also in $[0,1]$, order $H=H /(2 \pi)$ conversion.

\section{Robert Algorithm}

\subsection{Robert Algorithm}

Robert algorithm belongs to statistic texture description; its main idea is one derivative $\frac{\partial f}{\partial x}$ and $\frac{\partial f}{\partial y}$, the changes in the rate $x$ and $y$ direction of gray image. Set function $f(x, y)$, its derivative (gradient) for

$$
G(f(x, y))=\frac{\partial f}{\partial x} i+\frac{\partial f}{\partial y} j,
$$

Gradient mode is

$$
G(f(x, y))=\sqrt{\left(\frac{\partial f}{\partial x}\right)^{2}+\left(\frac{\partial f}{\partial y}\right)^{2}} .
$$

For digital image, type (5) is to the discrete form, so as to be difference operation to replace the differential operation. In the $\chi$ direction of the first order difference

$$
\Delta_{x} f(x, y)=f(x, y)-f(x+1, y),
$$

In the $y$ direction of the first order difference

$$
\Delta_{y} f(x, y)=f(x, y)-f(x, y+1)
$$

The gradient of the model can be simplified as

$$
\begin{aligned}
& G(f(x, y))= \\
& \sqrt{[f(x, y)-f(x+1, y)]^{2}+[f(x, y)-f(x, y+1)]^{2}},
\end{aligned}
$$

In order to facilitate the calculation of computer, usually use absolute value instead of type (8), i.e.

$G(f(x, y)) \approx|f(x, y)-f(x+1, y)|+|f(x, y)-f(x, y+1)|$.

Robert algorithm is

$$
\begin{aligned}
& G(f(x, y))= \\
& \sqrt{[f(x, y)-f(x+1, y+1)]^{2}+[f(x+1, y)-f(x, y+1)]^{2}},
\end{aligned}
$$

Type (10) with the absolute value approximated a

$$
\begin{aligned}
& G(f(x, y)) \approx \\
& \left|[f(x, y)-f(x+1, y+1)]^{2}+[f(x+1, y)-f(x, y+1)]^{2}\right|
\end{aligned}
$$

\subsection{Results of the Robert Algorithm Test}

In order to prove the effectiveness of the texture retrieval, design a set of the Robert algorithm for image retrieval. Because the Robert algorithm only using I component, without taking into account the $H$ and $S$ components. Figure 1 is submitted to the diagram, Figure 2 is sample the texture map of Robert algorithms using the Robert algorithm, and figure 3 shows the retrieval results.

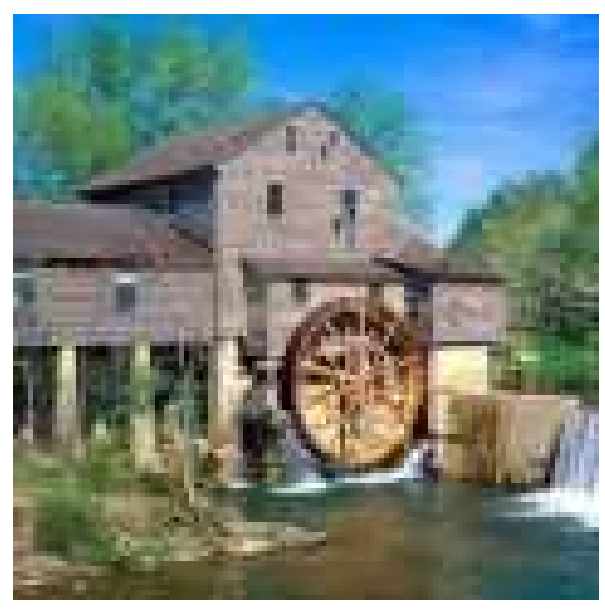

Fig. 1: Diagram

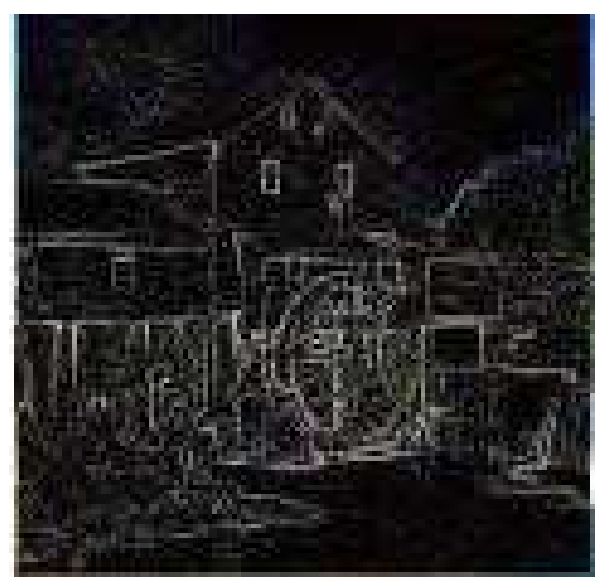

Fig. 2: sample texture map Robert algorithm processing

\section{Main Colour Projection Method}

\subsection{Main Value Extraction}

In this paper, using the algorithm, the fixed block image segmentation method. Firstly, the block is fixed, the 


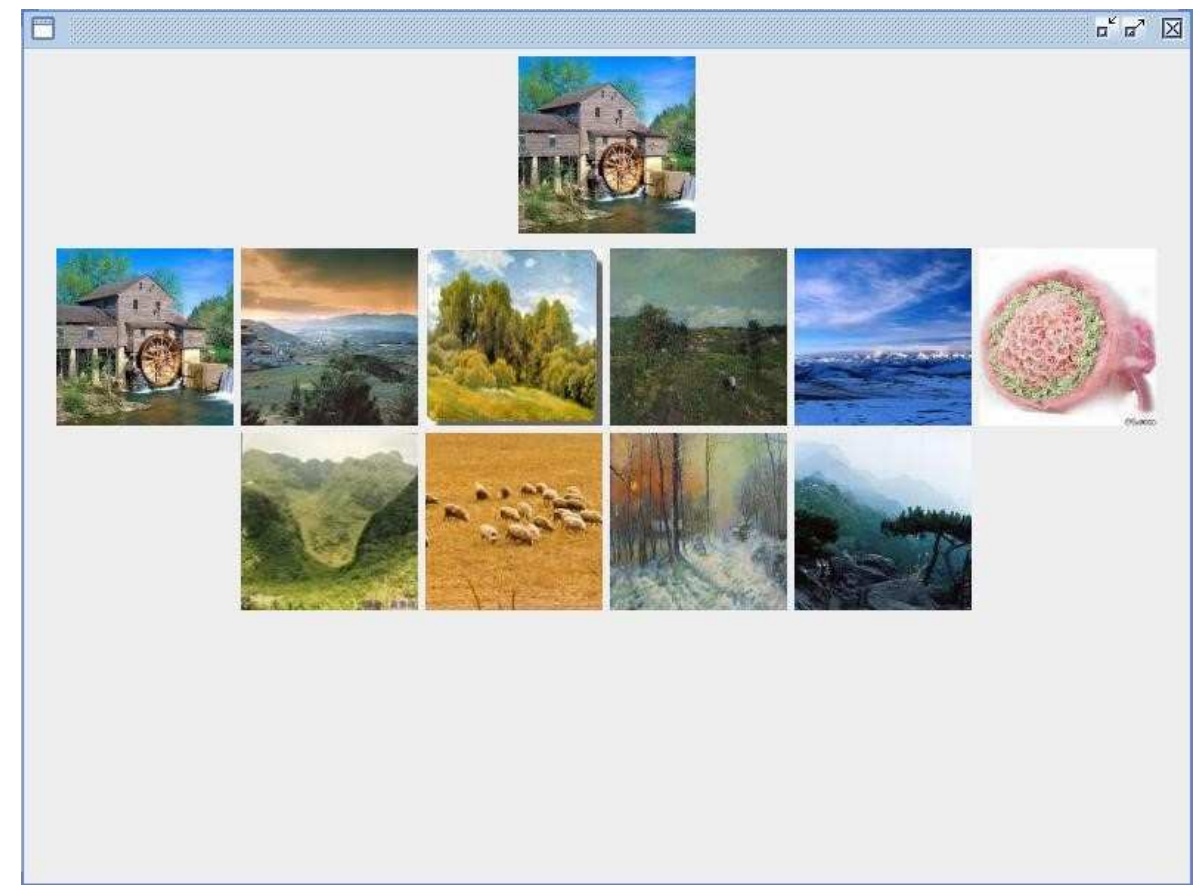

Fig. 3: Results for Robert algorithm

image divided into $n \times n$ with the same size grid. In order to describe color feature and gray feature of each block, this paper adopts the concept of value.

Definition 1 (main) for a characteristic block value (color or gray value), if it has the most number of pixels in the block, then the characteristic values (color or gray value) is defined as the block principal value. Let $M$ be the $i$ block main value, then:

$$
M_{i}=\left\{k \mid \operatorname{num}(i, k)=\max _{j=1}^{256}(\operatorname{num}(i, j))\right\}, \quad 1 \leq k \leq 256 .
$$

Among them, $j$ said the tone value (or gray), $\operatorname{num}(i, j) i$ said the tone of the block number of $j$ values of all pixels for the.

However, when the block is the image to the principal value is not may reflect the color feature and shape feature block, if the extracted principal value in the whole block contains a very small proportion of the value, main value is extracted is not enough to reflect the characteristics of the block, in order to eliminate the effect of this case the main value judgment, the following strategy to determine the extracted principal value as the block is class:

1.set threshold (threshold1), if $\operatorname{num}(i, k) /(m \times n)$

$\geq$ threshold1, $M_{i}=k$ is taken as the block principal value.

2.if $\operatorname{num}(i, k) /(m \times n)<$ threshold1 you need to consider the block near the block's main value to determine the block principal value, if the principal value of the adjacent block satisfy condition (1) and the block in the main value is equal to or less than a certain threshold of the difference (threshold1), $M_{i}=k$ is still set to the principal value, otherwise do not specify the main block. Among them, $m, n$ said, every block size. See Figure extraction value $4 \sim 7$ as shown.

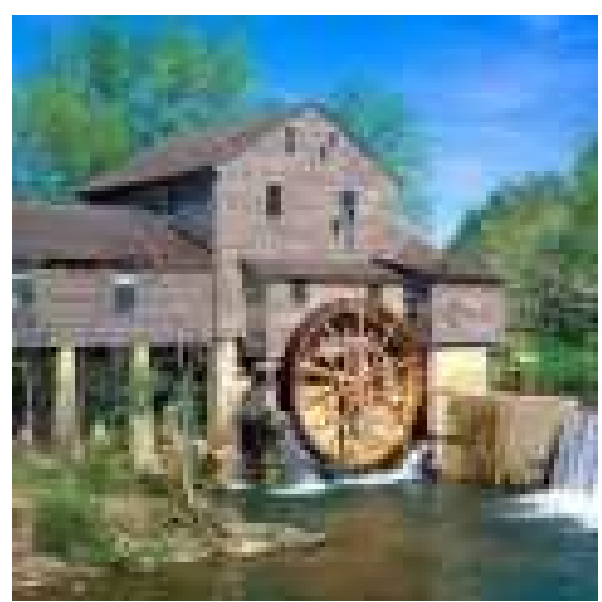

Fig. 4: Diagram 


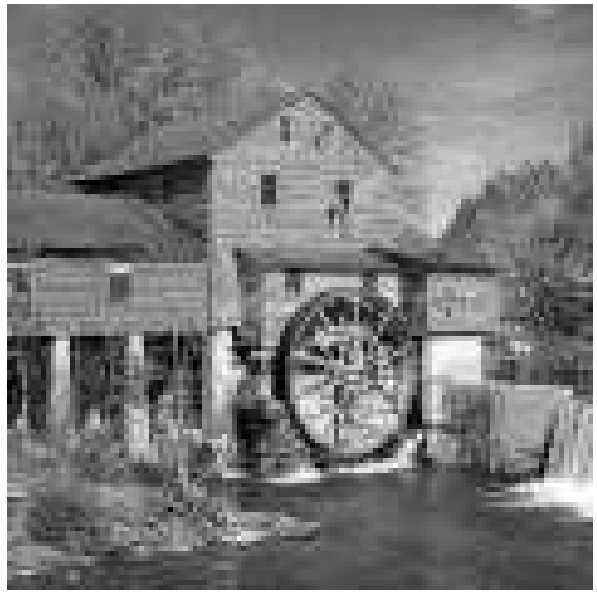

Fig. 5: an example of the grayscale

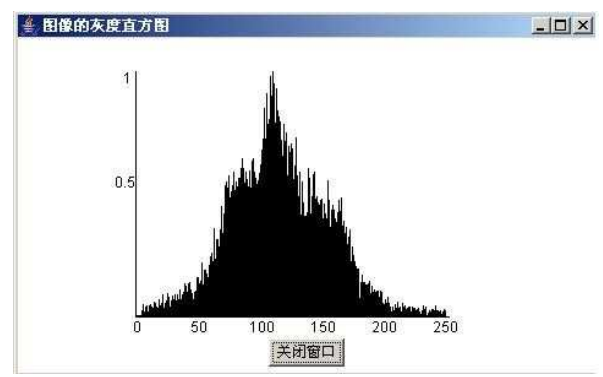

Fig. 6: gray-scale histogram

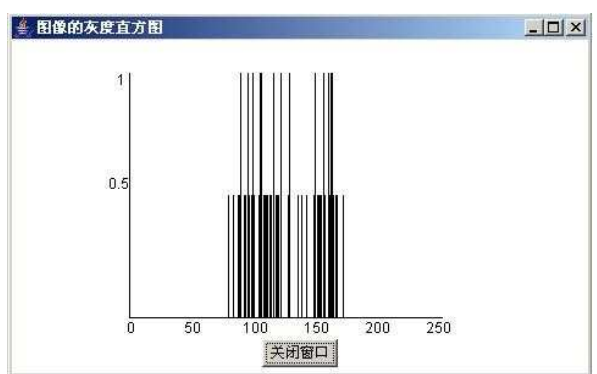

Fig. 7: Grey statistics histogram into extract the principal value

\subsection{Projection Histogram}

For a certain image features (such as component or components), with characteristics of the histogram, the histogram is a discrete function, i.e.:

$$
H(k)=\frac{n_{k}}{N}, \quad k=0,1, \cdots, L-1,
$$

In the type: characteristics of $k$ representative value of the image, $L$ are the number of feature values, $n_{k}$ are the number of pixels having characteristic values for the image, and $N$ are the total number of the image pixels.
Since the histogram is each pixel in an image of a statistical characteristic value of the frequency of occurrence of, only reflect the frequency of one image feature, spatial relationship without inclusion of image features, is applied to image retrieval, will reduce the accuracy of retrieval system.

In order to overcome the histogram does not reflect the image of a characteristic spatial distribution, this method in the statistical foundation block principal value using projection histogram. One feature of an image of each pixel are projected on the horizontal $x$ axis and the vertical $y$ axis, get the distribution of the features in the 2 direction. $H$ Projection component in the $x$ direction and $y$ direction reflects the distribution of the color image in space. For an image of the $H$ component in the $x$ direction and the $y$ direction of the projection histogram is defined as follows:

$$
\begin{aligned}
& P_{H}(x)=\frac{1}{h} \sum_{y=1}^{h} H(x, y), x=1,2, \cdots, w, \\
& P_{H}(y)=\frac{1}{w} \sum_{x=1}^{w} H(x, y), y=1,2, \cdots, h .
\end{aligned}
$$

Among them, $w$ and $h$ for the width and height of the image, $H(x, y)$ are the $H$ component images in $(x, y)$ pixel values.

\subsection{Feature Extraction}

Projection histogram of the image is discrete sequence; sequence length depends on the width and height of the image 8. Different image projection histogram processing, projection histogram will get different length. Based on the calculation of similarity of images is using the first three moment's projection histogram.

Central moment histogram shows the distribution histogram. On the first three moments of $P(i)$ histogram, respectively:

$$
\begin{gathered}
M_{1}=\frac{1}{L} \sum_{i=1}^{L} P(i), \\
M_{2}=\sqrt{\frac{1}{L} \sum_{i=1}^{L}\left(P(i)-M_{1}\right)^{2}} \\
M_{3}=\sqrt[3]{\frac{1}{L} \sum_{i=1}^{L}\left(P(i)-M_{1}\right)^{3}} .
\end{gathered}
$$

Using the HSI model of the $H$ components are projected to the $x$ direction and the $y$ direction, get 2 projection histogram is calculated respectively, $P_{H}(x)$ and $P_{H}(y)$ the first three moments of 2 histograms, 6 central moment. With 6 center distance as features to describe images, the image can be represented by a 7 dimensional vector $m$ :

$$
m=\left(M_{1}^{H x}, M_{2}^{H x}, M_{2}^{H x}, M_{1}^{H y}, M_{2}^{H y}, M_{3}^{H y}, R\right),
$$




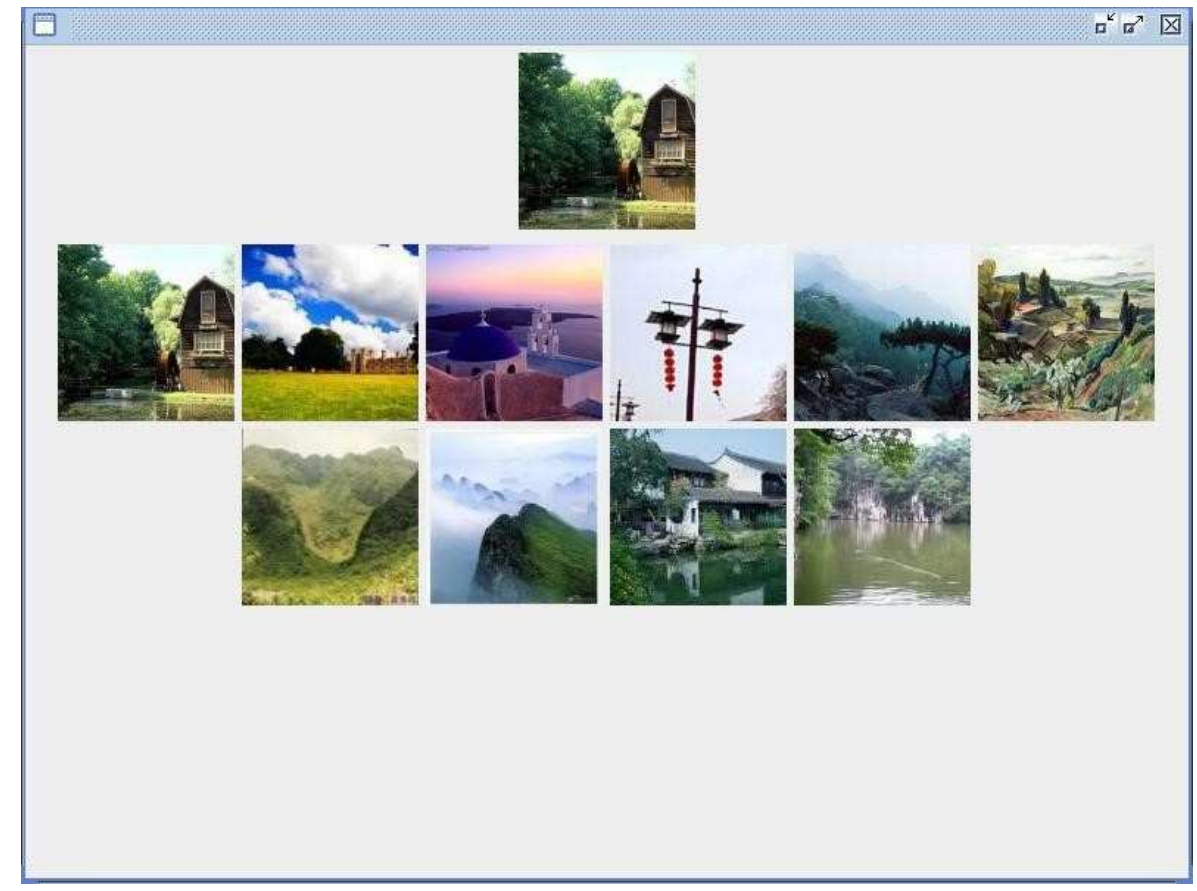

Fig. 8: retrieval of 10 pictures feedback

Among them, element $M_{1}^{H x}$ represents a central moments $H$ projection histogram in the $x$ direction of the image component, other elements in the vector meaning is similar, numerical R algorithm for Robert extraction.

\subsection{Similarity Calculation}

Let be $Q$ a query image, $D$ is an image in the database, they are available for above 7 dimensions, respectively, $q$ and $d$ said:

$$
\begin{aligned}
& q=\left(Q_{1}^{H x}, Q_{2}^{H x}, Q_{2}^{H x}, Q_{1}^{H y}, Q_{2}^{H y}, Q_{3}^{H y}, R_{q}\right), \\
& d=\left(D_{1}^{H x}, D_{2}^{H x}, D_{2}^{H x}, D_{1}^{H y}, D_{2}^{H y}, D_{3}^{H y}, R_{d}\right),
\end{aligned}
$$

The image $Q$ and $D$ the distance between $q$ the $d$ vector and is the distance between the weighted Euclidean distance to measure, as follows:

$$
\|q-d\|=\sqrt{W_{H} \sum_{i=1}^{3} \sum_{j=x, y}\left(Q_{i}^{H j}-D_{i}^{H j}\right)^{2}+W_{R} \sum_{l=1}^{n}\left(R_{q}-R_{d}\right)^{2}}
$$

Among them, $n$ is the number of pixels; $W_{H}, W_{R}$ weighting coefficient, is a group of experience, can be adjusted according to the test results. In this paper, $W_{H}=0.7, W_{R}=0.3$, because the $H$ component description ability and color visual approach, texture components reflect the image texture information in gray.

\section{Experimental Analysis}

In order to validate the effectiveness of the retrieval system, the author designed 3 sets of experiments, retrieval of the landscape for a class of image, image number 100, which contains similar to the target image has 5 beds, each query returns and the target image of 10 images most similar. Search results are shown in Figure 8

In Figure 8, the first row of the images for users to submit images, second and third row images are retrieval results analysis figure 8 shows:

1.due to the result according to the sorted result from small to large, from left to right, from top to bottom. Therefore, in Figure 8 the first image the second row of the target image.

2.Theoretically, in this paper is used the extraction of Robert numerical algorithm, principal value, projection histogram and weighted Euclidean distance, and in the weighted Euclidean distance, $W_{H}=0.7, W_{R}=0.3$, which makes the system on the $H$ component dependence. From the search results, we can be seen as the image submitted by users, the main color to blue and white, and in the global color retrieval results, the upper image searching out is mainly blue; at the same time, using the Robert algorithm, image target image texture search result from examination of the reflected. 


\section{Conclusion}

This paper presents an image texture and color image retrieval based on statistical projection, which can describe the color spatial distribution and texture information of the image, but the color spatial distribution information that is not very precise. Using this method not only can the redundant information filter color in the image, the image texture description, but also in the retrieval, calculation amount is small, fast speed, can also choose the features and different weights according to needs, how to represent the spatial distribution information of color, is the focus of future work.

\section{Acknowledgement}

The work is supported by the fund of China Academy of Tropical Agricultural Sciences postdoctoral foundation.

\section{References}

[1] Smith J R. Color for image retrieval [A]. Casteli V,Bergman L D,eds. Image Databases-Search and Retrieval for Digital Imagery [C]. John Wiley \& Sons, (2002).

[2] Swain M J,Ballard D H. Color indexing [J]. International Journal of Computer Vision, 7, 11-32 (1997).

[3] Zhang Y J,Yao Y R,He Y. Color image segmentation based on HIS Model [J]. Heigh Technology Letters, 4, 28-31 (1998).

[4] Beeze-Yates R,Ribeiro-Neto B. Modern Information Retrieval [M]. Addison Wesley, (1999).

[5] V N Gudivada, V V Raghavan. Content based image retrieval systems [J]. IEEE Computer, 28, 18-22 (1995).

[6] A Yoshitaka,T Ichikawa. A survey on content-based retrieval for multi-media databases [J]. IEEE Transaction on Konwledge and Data Enginneering, 11, 81-93 (1999).

[7] Chen Xinghua. The evolution of agricultural informationization and countermeasures $[\mathrm{J}]$. Science and technology management research, 7, 437-03 (2009).

[8] Cao Yong. To speed up the agricultural informationization construction to promote agricultural modernization development $[\mathrm{J}]$. Journal of Inner Mongolia science and technology and economy, 18, 63-64 (2009).

[9] Zhao chun. Henan province rural informatization way and strategy analysis $[\mathrm{J}]$. Journal of management informationization in China, 12, 81-83 (2009).

[10] Du Xulin Zhu Qin, Wen Huaiyu. New rural informationization present situation and development countermeasures [J]. Rural economy, 95-98 (2009).

[11] HUANGSH, SHEORANSK, WANGG. A review and analysis of supply chain operations reference (SCOR) model [J]. Supply Chain Management, 9, 23-29 (2004).

[12] CHOITY, DOOLEYKJ. Supply networks and complex adaptive systems: control versus emergence [J]. Journal of Operations Management, 19, 351-366 (2001).

[13] Zhang Ailing. What time thinking about the construction of speed up agricultural informationization [J]. Journal of agricultural science and technology, 17-18 (2007).

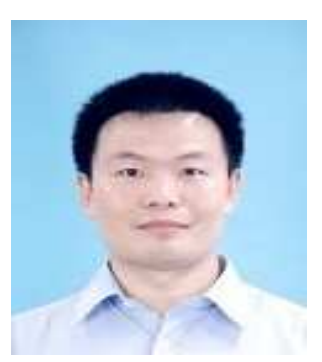

Xiaofei
Zheng
received PH.D in College
of Agriculture at Hainan
University. He is Assistant
Professor of the Institute of
Tropical Agricultural Science
and Technology Information
of CATAS. His research
interests are of agricultural
economics, agricultural informatization, germplasm resources development, agricultural strategy \& policy, global agricultural development, etc. He has published two academic monographs "Economics Study of Wild Rice Germplasm in Hainan" and "Study on Multilevel Development for Agricultural Informatization in Hainan". He has published more than 20 research manuscripts in different scientific journals,such as China Informatization, China Tropical Agriculture, Agricultural Economics Research, Hubei Agricultural Science, Ecological Economy, China Study Journal of Malaya University, etc.And two articles indexed by The Science Citation Index, five articles indexed by The Engineering Index.

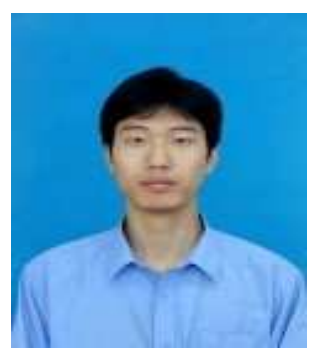

Zhe Gao received MEng in College of Information Science \& Technology at Hainan University. His research interests are of image processing, content-based image retrieval. He has published research article in Natural Science Journal of Hainan University.

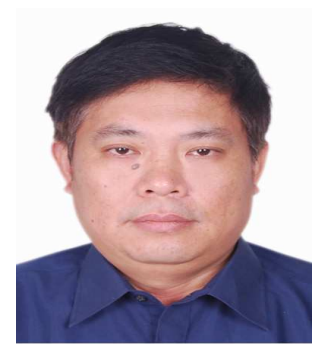

Enping Liu born in March 1965, professor, master tutor, mainly engaged in tropical agricultural economy, tropical agricultural industry information monitoring, and early warning. In recent years, presided over 10 research projects of the provincial and ministerial, published more than 30 papers, and edited a book. Have Hainan Provincial Science and Technology Progress Award and 3 Software copyrights. 